\title{
Pylephlebitis: A Diagnostic Challenge
}

\author{
Ana Machado $^{\mathrm{a}, \mathrm{b}}$, Sofia Ribeiro ${ }^{\mathrm{a}}$, Teresa Mendonca ${ }^{\mathrm{a}}$
}

\begin{abstract}
Pylephlebitis is a life-threatening condition commonly associated with diverticulitis in adults. The diagnosis is often missed because of its non-specific clinical presentation. We present the case of a 74-year-old woman that was admitted with abdominal pain and fever. Computerized tomography revealed thrombosis of the portal vein system and diverticulitis of the sigmoid colon. The patient was treated with antibiotics and anticoagulation. No thrombophilic risk factors were diagnosed.
\end{abstract}

Keywords: Pylephlebitis; Portal vein thrombosis; Acute diverticulitis; Cavernous transformation of mesenteric and splenic veins

\section{Introduction}

Pylephlebitis or septic thrombophlebitis of the portal vein is a rare entity with significant morbidity and mortality [1]. It is often a complication of intra-abdominal infection such as diverticulitis (most common), acute appendicitis, necrotizing pancreatitis, acute cholecystitis, colitis, inflammatory bowel disease, viscus perforation, hemorrhoidal disease, Behcet's syndrome or amebic colitis [2]. However, we cannot identify a primary source of infection in $70 \%$ of patients [3]. It can occur in all ages, but it seems to be more frequent in male (68-74\%) [3].

Because of its non-specific clinical and laboratory presentation, the diagnosis can be delayed [3], which usually worsens the prognosis of the disease. The definitive diagnosis requires demonstration of portal vein thrombosis, usually accompanied by bacteremia and fever [4].

The treatment consists in aggressive antibiotic therapy and surgical drainage of the infection focus, when it exists. Anticoagulation therapy is also an option but remains controversial [2].

We report a case of pylephlebitis due to an acute diverticulitis.

Manuscript submitted March 29, 2018, accepted April 19, 2018

aDepartment of Internal Medicine, Centro Hospitalar do Porto, Porto, Portugal ${ }^{b}$ Corresponding Author: Ana Machado, Department of Internal Medicine, Centro Hospitalar do Porto, Porto, Portugal.

Email: anamachado062@gmail.com

doi: https://doi.org/10.14740/jmc3050w

\section{Case Report}

A 74-year-old women was presented in the emergency department with a 20-day of abdominal pain and fever. She denied any recent travel or sick contacts. Her past medical history included colon diverticulosis and no chronic medication. She denied having any hypercoagulable workup done in the past, and denied any personal or family history of thrombosis or any hypercoagulable states.

On presentation, she appeared to be acutely ill and she had fever $\left(38.2^{\circ} \mathrm{C}\right)$. The remaining examination was unremarkable with the exception of the mild tenderness on deep palpation of the left flank of the abdomen, but no guarding or rebound tenderness. The spleen and liver were non-palpable.

Her blood panel showed 7,000 $\mu \mathrm{L}$ of leukocytes (normal 4 - 11,000/ $\mu \mathrm{L}$ ), C-reactive protein of $238 \mathrm{mg} / \mathrm{dL}$ (normal < $5 \mathrm{mg} / \mathrm{dL}$ ), creatinine $0.63 \mathrm{mg} / \mathrm{dL}$ (normal $0.5-0.9 \mathrm{mg} / \mathrm{dL}$ ), urea 16 ? $\mathrm{mg} / \mathrm{dL}$ (normal $10-50 \mathrm{mg} / \mathrm{dL}$ ), total bilirubin 0.49 $\mathrm{mg} / \mathrm{dL}$ (normal $0.2-1 \mathrm{mg} / \mathrm{dL}$ ), direct bilirubin $0.23 \mathrm{mg} / \mathrm{dL}$ (normal $0-0.2 \mathrm{mg} / \mathrm{dL}$ ), alkaline phosphatase $71 \mathrm{U} / \mathrm{L}$ (normal 32 - $104 \mathrm{U} / \mathrm{L}$ ), aspartate aminotransferase $14 \mathrm{U} / \mathrm{L}$ (normal 10 - $30 \mathrm{U} / \mathrm{L}$ ), alanine aminotransferase $9 \mathrm{U} / \mathrm{L}$ (normal 10 - 36 U/L) and gamma glutamyltransferase 29 U/L (normal 6 - 39 $\mathrm{U} / \mathrm{L}$ ). Prothrombotic screening test (protein $\mathrm{C}$, protein $\mathrm{S}$ and antithrombin III activity, factor VIII, IgM and IgG anticardiolipin antibodies, lupus anticoagulant and plasma homocysteine) were in normal range. Serological tests for HIV, syphilis, A, B and C hepatitis were negative. Blood cultures were negative too.

Thorax X-ray was unremarkable, but the abdominal ultrasound revealed mild splenomegaly and biliary lithiasis, with no signs of complication. A computerized tomography (CT) scan revealed thrombosis of the portal vein system (Fig. 1) and diverticulitis of the sigmoid colon (Fig. 2). Colonoscopy was inconclusive because of intolerance of the patient.

A conservative medical treatment approach was preferred and the patient started antibiotic therapy with piperacillin/tazobactam and anticoagulation with enoxaparin with good clinical evolution. She was discharged after 2 weeks asymptomatic and the antibiotic was switched to ciprofloxacin and metronodazole for 4 more weeks with total recovery. The anticoagulant therapy was replaced by dicumarinic without complications.

The patient remained asymptomatic and repeated CT 6 months after discharge. It demonstrated loss of definition of the superior mesenteric vein with an extensive collateral circulation. She remained asymptomatic and the CT, 6 months after the last one, demonstrated she had cavernous transformation of superior mesenteric and splenic veins. She maintained 


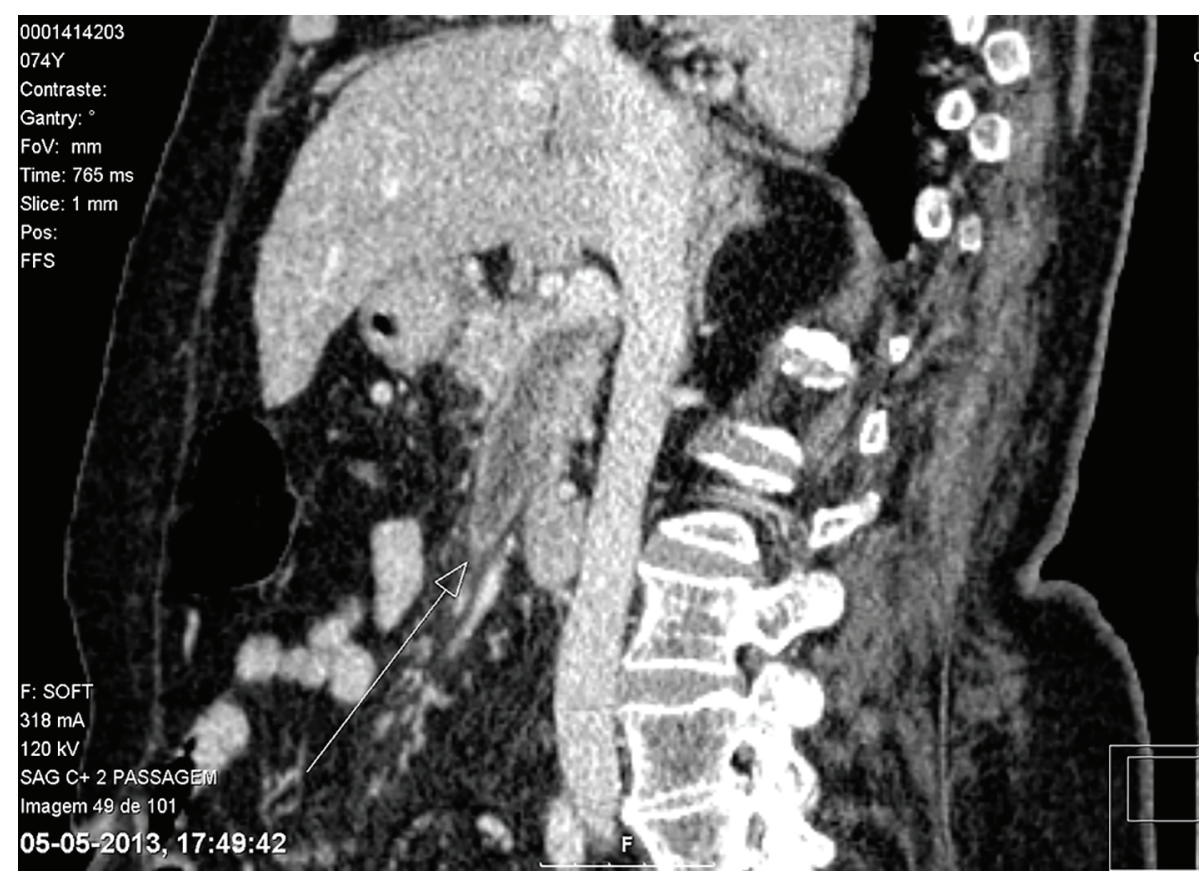

Figure 1. In the portal venous phase, there is a defect of repletion of the superior mesenteric vein and the portal vein, translating thrombosis.

anticoagulation.

\section{Discussion}

Diverticular disease is the main cause of pylephlebitis, as we could see in our case. However, a primary source of infection is not identified in $70 \%$ of patients [3].

It is usually polymicrobial [5], but the most common pathogens are Escherichia coli (54\%) and Proteus mirabilis $(23 \%)$ [1]. The thrombus spreads to the largest vessels, leading to septic thrombophlebitis of the mesenteric vein and, in

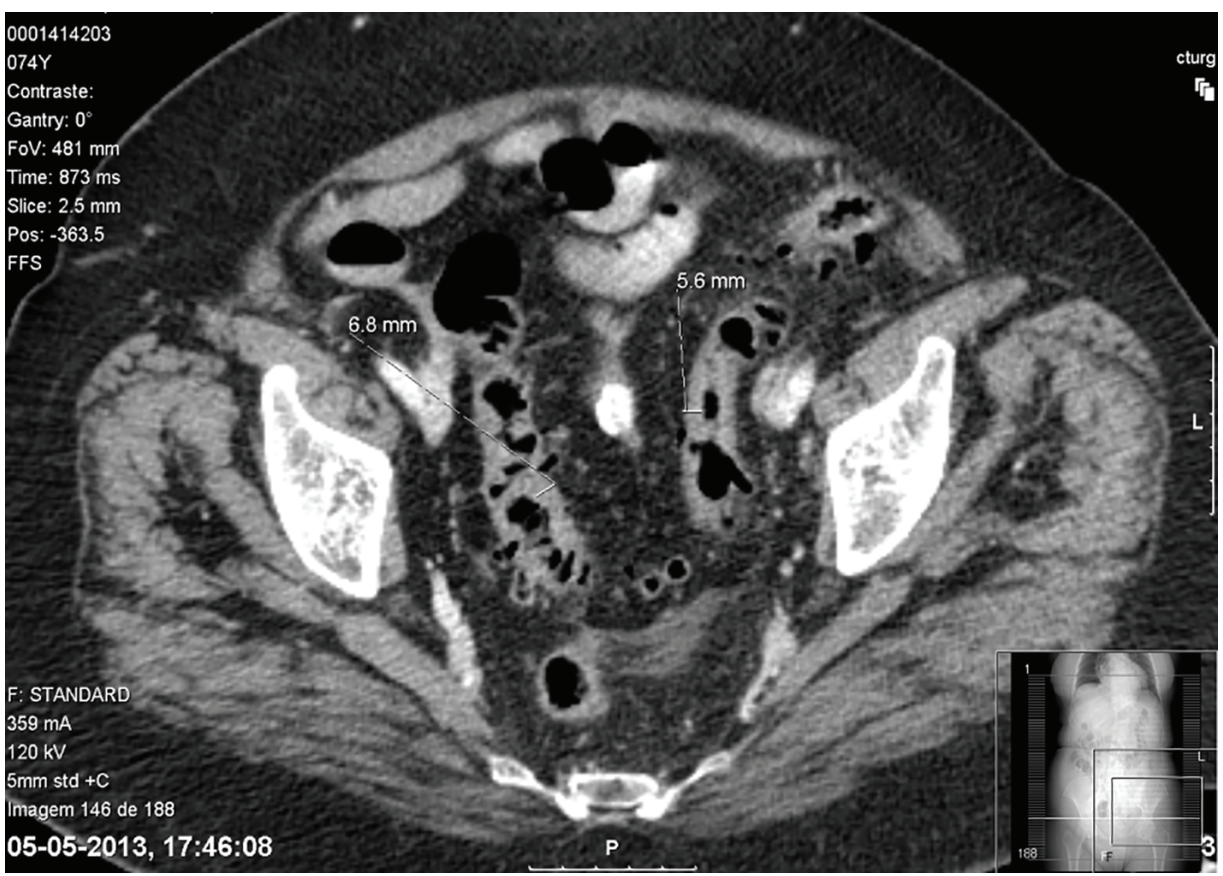

Figure 2. Concentric and segmental parietal thickening of the sigmoid colon, which presents multiple images of diverticulosis, associated with densification of pericolic fat, aspects translating acute diverticulitis. 
some cases, of the portal vein. The resulting bowel ischemia and infarction are associated with a high morbidity and mortality [6].

Clinical presentation is usually variable and non-specific, making diagnosis difficult [6], and mortality rate is still high $(25 \%)$ [3]. It can be asymptomatic or present with gastrointestinal symptoms (ex: abdominal pain, nausea, vomiting, and diarrhea), fever and constitutional symptoms (ex: fatigue, malaise, and anorexia/weight loss) [3]. We can also find hepatomegaly with elevated liver enzymes and jaundice [3], when associated with liver abscess or cholangitis [2]. Laboratory tests usually demonstrate elevated markers of inflammation [2]. Despite bacteremia has been reported in $50-80 \%$ cases, in our case we did not find the etiological agent [4].

Doppler ultrasound and contrast-enhanced CT facilitate early diagnosis: both can show portal vein thrombosis and signs of the primary abdominal inflammatory process, but accuracy of ultrasound is limited by the interference of bowel gas and by the operator [6]. FDG-PET can be a useful imaging technique to diagnose pylephlebitis, but sensitivity and specificity are variable in different studies [4].

The diagnosis can be considered with the demonstration of portal vein thrombosis in a febrile patient with bacteremia [4].

In our case, the absent of bacteremia do not exclude the diagnosis, considering it may not exist in $20-50 \%$ of the cases.

Treatment of pylephlebitis consists on treating the primary septic process by using broad-spectrum antibiotics to cover for enteric facultative gram-negative bacilli, anerobic and aerobic Streptococcus species [7]. The duration of therapy is controversial, but the majority of authors recommend a minimum of 4 weeks of treatment [3]. Adequate surgical intervention may be needed [6]. The anticoagulation therapy is controversial, but the available data are limited. Some authors consider that it prevents thrombus propagation, reduces the risk of mesenteric ischemia, improves recanalization and reduces the risk of recurrent thrombosis [2].

The prognosis depends, not only on the patient inherent characteristics and the severity of the clinical presentation, but also on the precocity of the diagnosis and treatment initiation. Mortality usually results from severe sepsis secondary to an overwhelming intra-abdominal infection [3].

It can result in full recovery, but there have been described cases of cavernomatous transformation of the portal vein and portal hypertension [6].

\section{Conclusion}

Pylephlebitis is a rare condition characterized by a non-specific presentation. That is why mortality remains high, despite the advances in diagnosis techniques. A high index of suspicion is required for making an early diagnosis. The timely administration of broad-spectrum antibiotics appears to be the most critical component of pylephlebitis treatment. Ideal time of antibiotic therapy and the benefits and duration of anticoagulation need further examination.

\section{Conflict of Interest}

The authors declare there is no conflict of interest.

\section{Funding Source}

There is no funding source.

1. Van De Wauwer C, Irvin TT. Pylephlebitis due to perforated diverticulitis. Acta Chir Belg. 2005;105(2):229230.

2. Levin C, Koren A, Miron D, Lumelsky D, Nussinson E, Siplovich L, Horovitz Y. Pylephlebitis due to perforated appendicitis in a teenager. Eur J Pediatr. 2009;168(5):633635.

3. Wong K, Weisman DS, Patrice KA. Pylephlebitis: a rare complication of an intra-abdominal infection. J Community Hosp Intern Med Perspect. 2013;3(2).

4. Mannaerts L, Bleeker-Rovers CP, Koopman M, Punt CJ, van Herpen CM. Pylephlebitis after a duodenal ulcer in a patient with metastasised colon carcinoma treated with chemotherapy and bevacizumab: a case report. Neth J Med. 2009;67(2):69-71.

5. Hagopian T, Zuniga F, Surani SR. Pylephlebitis: an uncommon complication of intra-abdominal infection. West J Emerg Med. 2011;12(4):575-576.

6. Castro R, Fernandes T, Oliveira MI, Castro M. Acute appendicitis complicated by pylephlebitis: a case report. Case Rep Radiol. 2013;2013:627521.

7. Lim HE, Cheong HJ, Woo HJ, Kim WJ, Kim MJ, Lee $\mathrm{CH}$, Park SC. Pylephlebitis associated with appendicitis. Korean J Intern Med. 1999;14(1):73-76. 